\title{
An aerothermodynamic design optimization framework for hypersonic vehicles
}

\author{
Simone Di Giorgio ${ }^{\mathrm{a}}$, Domenico Quagliarella ${ }^{\mathrm{b}}$, Giuseppe Pezzella ${ }^{\mathrm{c}, *}$, \\ Sergio Pirozzolia \\ ${ }^{a}$ Dipartimento di Ingegneria Meccanica e Aerospaziale, Sapienza Universitá di Roma, via \\ Eudossiana 18,00184 Roma, Italy \\ ${ }^{b}$ Multidisciplinary Analysis and Design Laboratory. Italian Aerospace Research Centre. \\ CIRA. Via Maiorise, I-81043. Capua (CE), Italy. \\ ${ }^{c}$ Analysis and Extrapolation to Flight Laboratory. Italian Aerospace Research Centre. \\ CIRA. Via Maiorise, I-81043. Capua (CE), Italy.
}

\begin{abstract}
In the aviation field great interest is growing in passengers transportation at hypersonic speed. This requires, however, careful study of the enabling technologies necessary for the optimal design of hypersonic vehicles. In this framework, the present work reports on a highly integrated design environment that has been developed in order to provide an optimization loop for vehicle aerothermodynamic design. It includes modules for geometrical parametrization, automated data transfer between tools, automated execution of computational analysis codes, and design optimization methods. This optimization environment is exploited for the aerodynamic design of an unmanned hypersonic cruiser flying at $M_{\infty}=8$ and $30 \mathrm{~km}$ altitude. The original contribution of this work is mainly found in the capability of the developed optimization environment of working simultaneously on shape and topology of the aircraft. The results reported and discussed highlight interesting design capabilities, and promise extension to more challenging and realistic integrated aerothermodynamic design problems.
\end{abstract}

Keywords: Hypersonics; Design optimization; Evolutionary strategies; CST.

\footnotetext{
${ }^{*}$ Corresponding author.

E-mail address: g.pezzella@cira.it
} 


\section{Introduction}

The evolution and the improvements in aerospace sciences have been constantly driven by the goal of flying faster and higher. Current applications of hypersonic flight mainly pertain to space flight activities, although extensions to passenger transportation are also envisaged. With this in mind, the present research effort focuses on the optimization-based aerothermodynamic design of hypersonic aircraft. For that purpose, an integrated design environment has been developed which includes in-house developed, open-source software and research codes, to provide an integrated optimization loop for shape design of hypersonic vehicles. For instance, the present optimization framework features modules for geometric parametrization, automated data transfer between analysis tools, automated execution of computational analysis code and design optimization methods. The Covariance Matrix Adaptation Evolutionary Strategy (CMA-ES [1]) is used to address aeroshape optimization. The original contribution of this work can be mainly found in the capability of the developed optimization environment of working, simultaneously, on shape and topology of the aircraft. Indeed, the parametrization is based on a special formulation of the class/shape function transformation method (CST) [2, 3, 4], which allows seamless and efficient design of both winged and lifting body configurations.

In general sense, topology optimization deals with the design of aeroshapes that are not classifiable in a particular class or type. In structural optimization, for example, the design of a wing or fuselage can be split into two phases, namely the definition of the general part layout with stringer and frames according to a given criterion or layout, and, subsequently, the sizing of such structural elements in order to achieve the required performance in terms of weight and strength. Most advanced approaches to topology optimization are able to perform both layout change and structural element sizing at the same time. The approach proposed here, although simplified and not as powerful and general as a Level Set Method [5], addresses the same class of problems, in the sense that the aerodynamic and aerothermodynamic performances of the shapes under investigation change dramatically if the shape is winged or not, and the optimal configuration heavily depends on the design conditions. Since a full level set approach presents a series of problems and hindrances when applied to our class of problems, we figured out an alternative approach that allows to change the configuration under investigation with continuity from a wingless to a winged shape. As said before, this goal 
is achieved by using a special CST parameterization. The advantage of this choice is that a very compact parameterization can describe smooth surfaces (as required by our solvers and by the problems under investigation) and, at the same time, different configuration classes. As a consequence, the output of the optimization will result in a winged or wingless body in function of the particular problem that is undertaken. In this framework, the optimization environment is exploited for the aerodynamic design of an unmanned hypersonic cruiser able to perform experimental activities flying at $M_{\infty}=8$ and $30 \mathrm{~km}$ altitude. Note that, this flight condition is the one commonly expected for a Hypersonic Speed Civilian Transportation (HSCT). Examples of HSCT, under development by the European Space Agency within the Seventh Framework Program are the HEXAFLY and HEXAFLY-INT vehicle concepts $[6,7]$.

\section{Overview of aeroshape optimization of hypersonic vehicles}

The problem of hypersonic vehicle aeroshape optimization has been addressed in several previous publications. Bowcutt et al. [8] reported the studies for hypersonic vehicles using the numerical optimization with the goal of maximizing the lift-to-drag ratio $(L / D)$ of a waverider vehicle. They prove that the optimized configuration exceeds the $L / D$ barrier defined by Kuchemann [9]. The same author also analyzed the coupling of aerodynamic and propulsive performances for a hypersonic cruise [10]. In this work, the multi-disciplinary optimization approach was used to design a simple hypersonic aircraft able to accomplish a cruise flight at Mach 15 with maximum $L / D$. The propulsive, aerodynamic, control, and stability design issues were analyzed as standard simple components of the aircraft. Constrained optimization of three-dimensional aeroshapes was assessed by Sheffer and Dulikravich [11]. In that work, the design variables were the coordinates of the vehicle's cross sections, the constraints were the useful vehicle volume and the length, and the objective was the aerodynamic drag reduction.

Landon et al. [12] developed a software to design optimal aeroshapes according to accurate and realistic design objectives and constraints. The software used a series of tools to predict geometrical features, mass properties, aerodynamic database and control and stability performances. In particular, it used the free-form deformation method for geometric parametrization and the supersonic/hypersonic arbitrary body program (S/HABP [13]) to evaluate vehicle aerodynamics and aerothermodynamics. A different approach 
was practiced by Ferguson et al. [14]. Here, the inverse design approach was used to generate the vehicle aeroshape once known the flowfield. The aim of that work was to find a feasible waverider-like vehicle with maximum $L / D$. A multidisciplinary design optimization (MDO) was developed by Bowcutt [15] which combines propulsion and external aerodynamic forces, mass properties and internal volumetric modeling, with the objective to maximize overall mission range of a hypersonic cruise missile. In the work of Starkey et al., an air-breathing waverider-based vehicle was optimized considering the propulsive system, the aerodynamic performance and hypersonic cruise trajectory [16]. CFD-based shape optimization of hypersonic vehicle was carried out by [17]. The aerodynamic performance was evaluated by means of inviscid CFD results and the objective of this optimization analysis is to attain maximum $L / D$ in the transonic and hypersonic flow regimes. Another example of multi-objective optimization can be found in Dominic and Erwin [18], where a capsule and a winged re-entry vehicles were optimized. The objective of this aeroshape optimization is related to mass, volume and re-entry range.

Multidisciplinary design optimization of the NASA HL-20 concept was carried out by Lobbia [19]. In particular, the goal was maximizing the aerodynamic efficiency assuming the re-entry heating as a constraint. Finally, Iuliano and Quagliarella [20] provided highlights about modern optimization techniques based on multi-fidelity and surrogate based optimization algorithms. The above research efforts are justified by the fact that the design of a hypersonic aircraft is extremely challenging as aerodynamic, control, propulsion and structure topics are highly connected each other, thus greatly influencing each other. Hence, it is expected that synergistic interactions, between vehicle sub-systems and functions, can produce significant performance and economic benefits. With this in mind, the design approach must pass from conventional design to a more complex and challenging highly integrated design (HID). However, a HID demands a series of advanced design tools and an increasing computational power, since a large number of design variables is usually involved. For this reason, numerical optimization is a fundamental tool in the integrated design process, which allows searching for the best solution that satisfies both requirements and constraints. In doing this, the present framework tool is able to efficiently build both winged and lifting body architectures in a seamless way, depending on the particular problem to be solved. This figure of merit of the tool will be illustrated in detail with the help of two test cases which, starting from the same parametrization, 
will lead to two different topologies. The driving force will be the constraints that, in one case put emphasis on reduce weight; while in the second one, on payload mass increase. It is worth noting that the optimal configuration will also shows changes in the aerodynamic shape. The advantages of this approach, even in a preliminary and exploratory design phase, are evidently in a more effective exploration of the design space. Indeed, a purely topological optimization, without changes in the aerodynamic shape, like wing or fuselage sections, might easily lead to sub-optimal configurations unable to fulfill all the problem constraints. On the other hand, pure shape change, without considering different topologies or configurations, is very useful in more mature design phases, but can only offer marginal advantages in preliminary and exploratory steps.

\section{Design optimization loop}

The optimization of a HID process requires several features as, for example, modularity of the design environment that is an important figure of merit. Generally speaking, the fundamental tools for numerical optimization are divided in two main classes, namely evaluators of objectives and constraints, and optimization softwares. The evaluators are packages, codes or functions able to evaluate both objective function and constraints that are function of design variables and are often linked with each other. Hence, the optimization software must be able to define objective and constraints as functions of the design parameters which are the problem variables. The software must also be able to handle constants like mission parameters such as cruise speed, angle of attack and aircraft altitude. Another essential feature is the capability of defining an initial set of solutions through smart parameter space sampling techniques. This feature is particularly useful when evolutionary optimizers are used and the quality of the initial population may influence the convergence towards the optimal solution. These capabilities are integrated in the present research effort, thus defining the optimization loop provided in Fig. 1, whereas Fig. 2 shows, in detail, the steps of population evaluator. The developed HID integrates several modules, such as geometric parametrization system, automated data transfer between analysis tools, automated execution of computational analysis code, design optimization methods, analysis of robust design optimization and analysis of uncertainty, grid computing for a rapid and high reliable analysis and validation of results. These modules define a robust and modular software framework able 


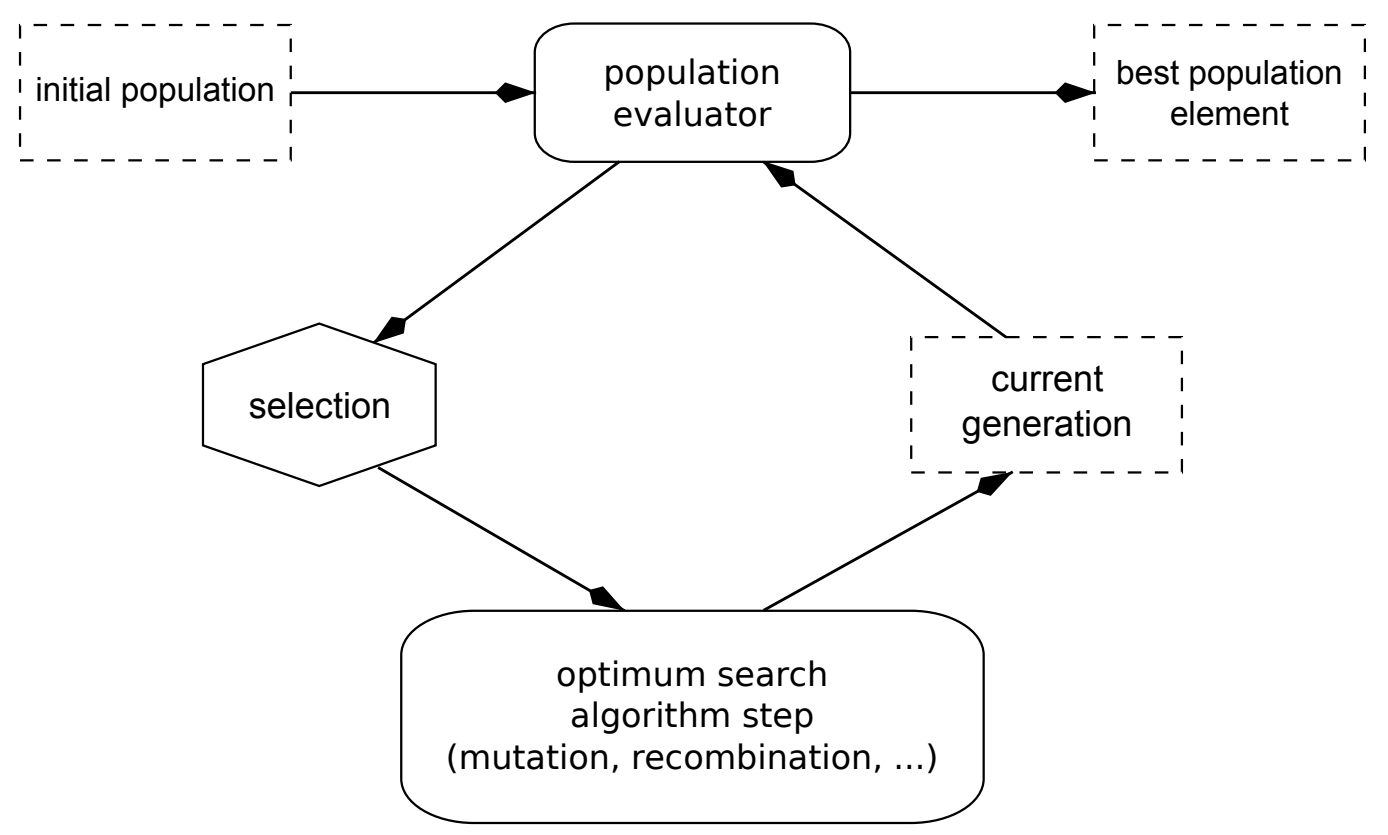

Figure 1: General numerical optimization flow chart.

to address a numerical optimization design. In particular, the computational tools include: the geometry package for aeroshape building, and the aerodynamic, the trajectory, and the thermal solvers. Details about geometry parametrization and aerodynamic, aerothermodynamic and flight mechanics tools are provided in the following sections. Finally, it is worth noting that the developed optimization framework features very interesting design capabilities as it allows working simultaneously on both shape and topology of the aircraft. The in-house developed ADaptive Genetic optimization library (ADG) is here used as optimization module [21].

\subsection{Geometrical parametrization}

Geometrical parametrization is a crucial ingredient in the design optimization procedure, and it requires a mathematical model/function to translate discrete design variables into the vehicle aeroshape. Several methods 


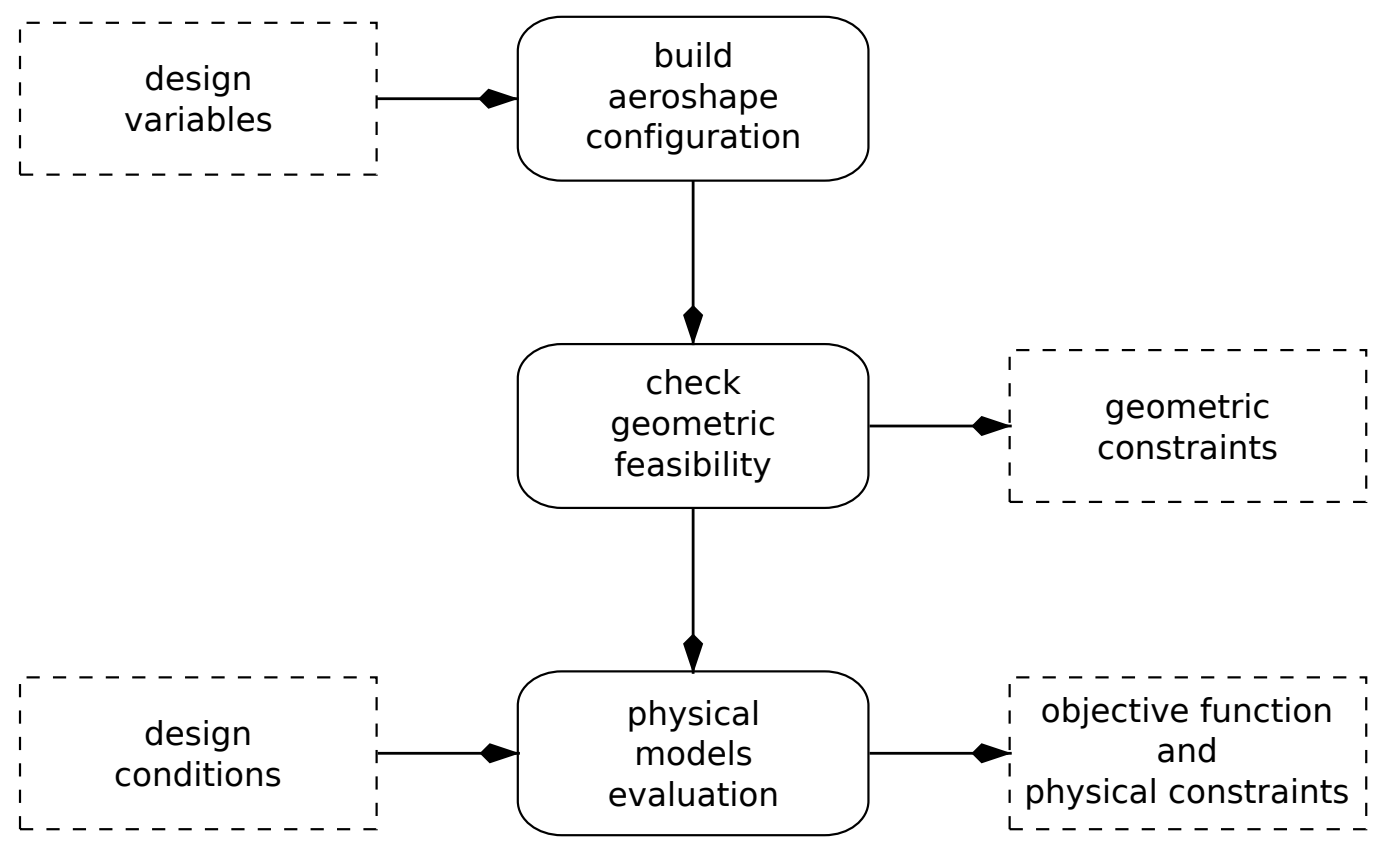

Figure 2: Population evaluator flow chart.

are available in literature, including Hicks-Henne functions [22], parametricsection airfoil parametrization [23], Bézier curves [23], nonuniform rational B-splines (NURBS) [24], CAD-based approaches [25], class/shape function transformation method (CST) [2, 3, 4], free-form deformation [26, 27, 28] and radial basis function approach [29].

In the present work the NURBS and the CST method have been integrated in the geometry package. The NURBS approach, widely used in computer graphics and in modern CAD-CAM-CAE software, relies on control points and nodes to generate parametric curves and surfaces, yielding considerable control and good shaping capabilities [30]. Application of NURBS to a typical lifting-body aeroshape was reported by Di Giorgio [31].

The CST method divides aircraft components into two classes of representation, namely Class 1 (wing/airfoil type shapes) and Class 2 (body cross-section type shapes), each defined through a suitable mathematical description, in terms of continuous but nonanalytic functions. 


\subsubsection{Wing representation}

The planform shape of the wing (see Fig. 3) is here assumed to consist of three straight chunks, having different sweep angle $\left(\lambda_{i}\right)$ changing at selected spanwise stations $\left(y_{i}\right)$, and with chord distribution defined by $c_{i}$. Following Kulfan and Bussoletti [2], Kulfan [3] the upper (U) and lower (L) surfaces of the wing are assumed in the form

$$
\zeta_{U, L}(\xi, \eta)=C_{N 2}^{N 1}(\xi) S_{U, L}(\xi)-\xi \tan \alpha+\eta \tan \beta
$$

where $\xi=\left(x-x_{L E}(\eta)\right) / c(\eta), \eta=2 y / b, \zeta=z / c(\eta)$ are the nondimensional streamwise, spanwise, and vertical space coordinates, $\beta$ is the wind dihedral angle, and $\alpha$ defines the wing angle with respect to the fuselage. All the wing profiles are assumed to be geometrically similar, and defined through a class function

$$
C_{N 2}^{N 1}(\xi)=\xi^{N 1}(1-\xi)^{N 2},
$$

where $N 1$ and $N 2$ determine the shape of the airfoil leading and trailing edge, and a shape function assumed to be a Bernstein polynomial of order $n$ (here $n=8$ ),

$$
S_{U, L}(\xi)=\sum_{i=0}^{n} A_{U, L_{i}} S_{i}(\xi)
$$

where

$$
S_{i}(\xi)=K_{i} \xi^{i}(1-\xi)^{n-i}, \quad K_{i}=\left(\begin{array}{c}
n \\
i
\end{array}\right)=\frac{n !}{i !(n-i) !} .
$$

To summarize, the wing is fully defined by specifying twenty-nine parameters, namely $\lambda_{i}(i=1-3), c_{i}(i=0-3), y_{i}(i=1-3), \alpha, \beta, N_{1}, N_{2}, A_{U, L_{i}}$, ( $i=0-8$, where for greater continuity at the leading edge we have set $\left.A_{U_{0}}=A_{L_{0}}\right)$, all of which are assumed to be design variables.

\subsubsection{Fuselage representation}

Again, following Kulfan and Bussoletti [2], the cross-sectional and the longitudinal shape of the fuselage are assumed to be the product of a class function and a shape function. Specifically, the cross-sectional shape is defined through the functions

$$
C c(\eta)=\eta^{N C}(1-\eta)^{N C}, \quad S c_{U, L}(\eta)=\sum_{i=0}^{n} A c_{U, L_{i}} \cdot S_{i}(\eta)
$$




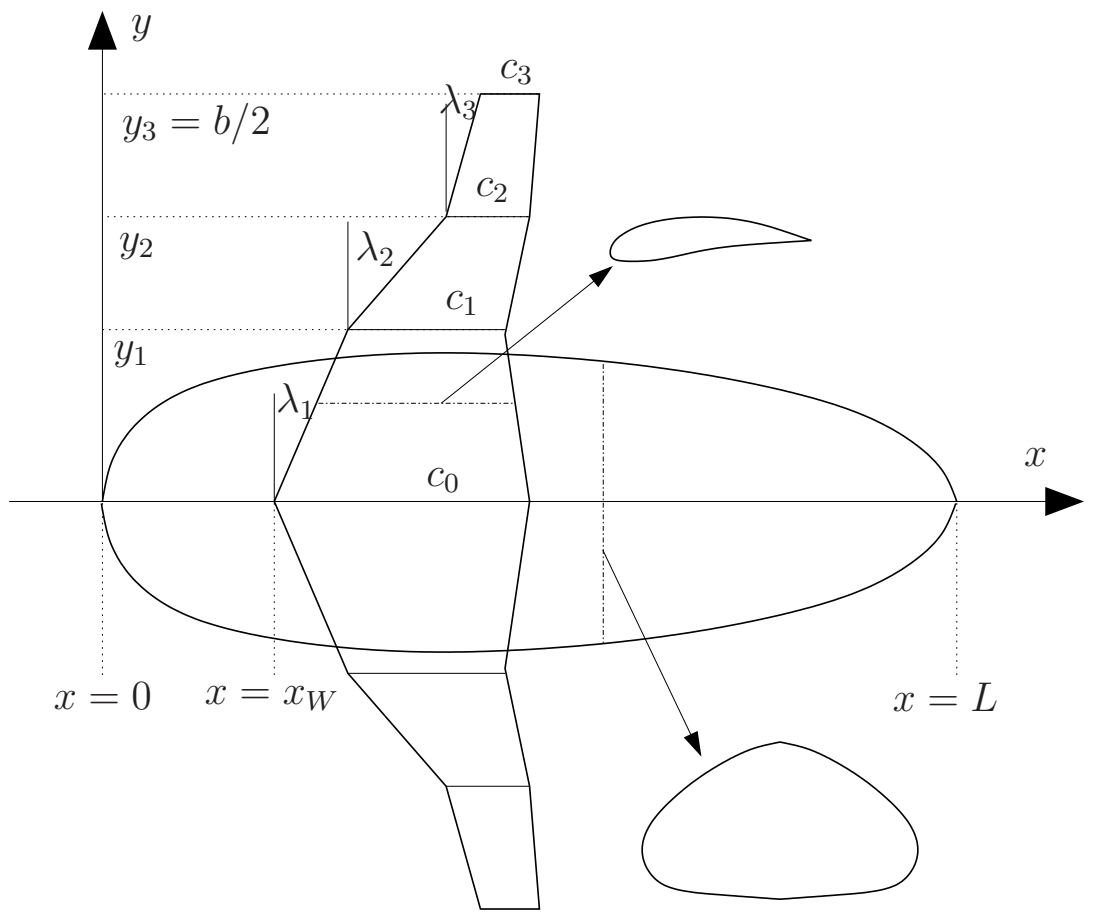

Figure 3: Sketch of a representative aircraft configuration. 
whereas the longitudinal shape is defined through the functions

$$
C d(\xi)=\xi^{N D 1}(1-\xi)^{N D 2}, \quad S d(\xi)=\sum_{i=0}^{n} A d_{i} \cdot S_{i}(\xi)
$$

where $\xi=x / L, \eta=y /(C d(\xi) S d(\xi))+1 / 2$ are the nondimensional streamwise and spanwise space coordinates, and the $S_{i}$ were defined in Eqn. (4). It should be noted that Kulfan and Bussoletti [2] assumed the $S c$ and $S d$ functions to be constant, whereas we allow for a more general representation in order to be able to obtain greater variety of aeroshapes, also including blended bodies. The upper and lower surfaces of the fuselage are accordingly given by

$$
z_{U, L}(\xi, \eta)=C c(\eta) S c_{U, L}(\eta) C d(\xi) S d(\xi) .
$$

If needed, the fuselage width and height may then be easily found as a function of the parameters defining the class and shape functions. To summarize, the fuselage is fully defined by specifying twelve parameters (i.e., design variables), namely $L, N_{C}, N_{D_{1}}, N_{D_{2}}, A c_{U, L_{i}}(i=0-3), A d_{i}(i=0-3)$ (hence with a Bernstein polynomial of order three), as symmetry about the $x-z$ plane requires $A c_{U, L_{i}}=A c_{U, L_{n-i}}$. Overall, geometrical characterization of the aircraft geometry requires specification of forty-two design variables, including the relative position of the wing with respect to the fuselage $x_{W}$ (see Fig. 3), all of which are here determined as a result of the optimization loop. The set of design variables further includes the wind incidence angle with respect to the fuselage axis, hereafter referred to as AoA, assuming symmetric flight conditions. Finally, note that above approach allows also obtaining blended body aeroshapes thanks to a continuous variation of the fuselsge shape.

\subsection{Models for Aerodynamics, Aerothermodynamics and Flight Mechanics}

The optimization-based design approach aims at improving the aerothermodynamic and aerodynamic performance of the vehicle by changing its shape. Further, it verifies the vehicle's ability to fly within the flight corridor, thus withstanding the related aero-thermal loading environment. As a result, the setup of an effective design optimization loop addresses contrasting problems, like high-fidelity computations and explorative capabilities of the optimization algorithm adopted in view of escaping local optima and approaching global ones. Our design problems require optimization algorithms with advanced exploring capabilities, like evolutionary algorithms, and therefore 
cannot utilize gradient-based techniques that take advantage of fast gradient computation techniques, like the adjoint-based solvers. With this in mind, the appraisal of vehicle aerodynamics relies on Newtonian panel methods for the inviscid flow analysis, whereas effects of friction are accounted through engineering-based methods [32]. For the sake of simplicity, high-temperature real gas effects are neglected. Indeed, as it is well known and documented in scientific literature, Newtonian aerodynamics offer an extremely good level of fidelity for the class of problems that we are interested in. Nevertheless, we cross-checked the obtained results using classical, more reliable fluid numerical computations and we found more than satisfactory result comparisons. Anyway, two different approaches are considered to account for viscous effects (friction and heat transfer). The first relies on methods available in the Viscous Effects On Complex Configurations code (VECC) which now includes S/HABP as a module, [33] for laminar and turbulent flow conditions, whereas the second relies on engineering relationships available for leading edges (fuselage, wing and tails) and flat plate configurations. The vehicle surface temperature is estimated with and without accounting for heat conduction inside the thermal protection system (TPS). In the former case, the conductive heat transfer through the TPS, which carries the energy towards the internal vehicle cold structure, is addressed also accounting for the cooling effect due to surface radiation. In the latter one, only radiation cooling applies at the surface of vehicle TPS. Finally, the vehicle trajectory is determined by solving the flight mechanics equations for a planar flight path.

\subsection{Optimization environment setup}

The optimization environment developed has been previously used for a typical re-entry mission [31], and it is hereafter applied to a hypersonic cruise mission. A vehicle shape is sought which optimizes the performance and satisfies design requirements and constraints. The design optimization loop is shown in Fig. 4. Once the bounds of design variables are defined, the optimization code writes two input files. These two files report the design variables of vehicle, which represent the main geometrical features of the aircraft. They also are the input parameters for the geometry generation package. The present framework tool reads the input files, generates the aeroshape of the sample vehicle and, then, writes the input file for the streamlines generator and the aerothermodynamics solver. After that the geometry package calculates all the aeroshape geometric features useful to 


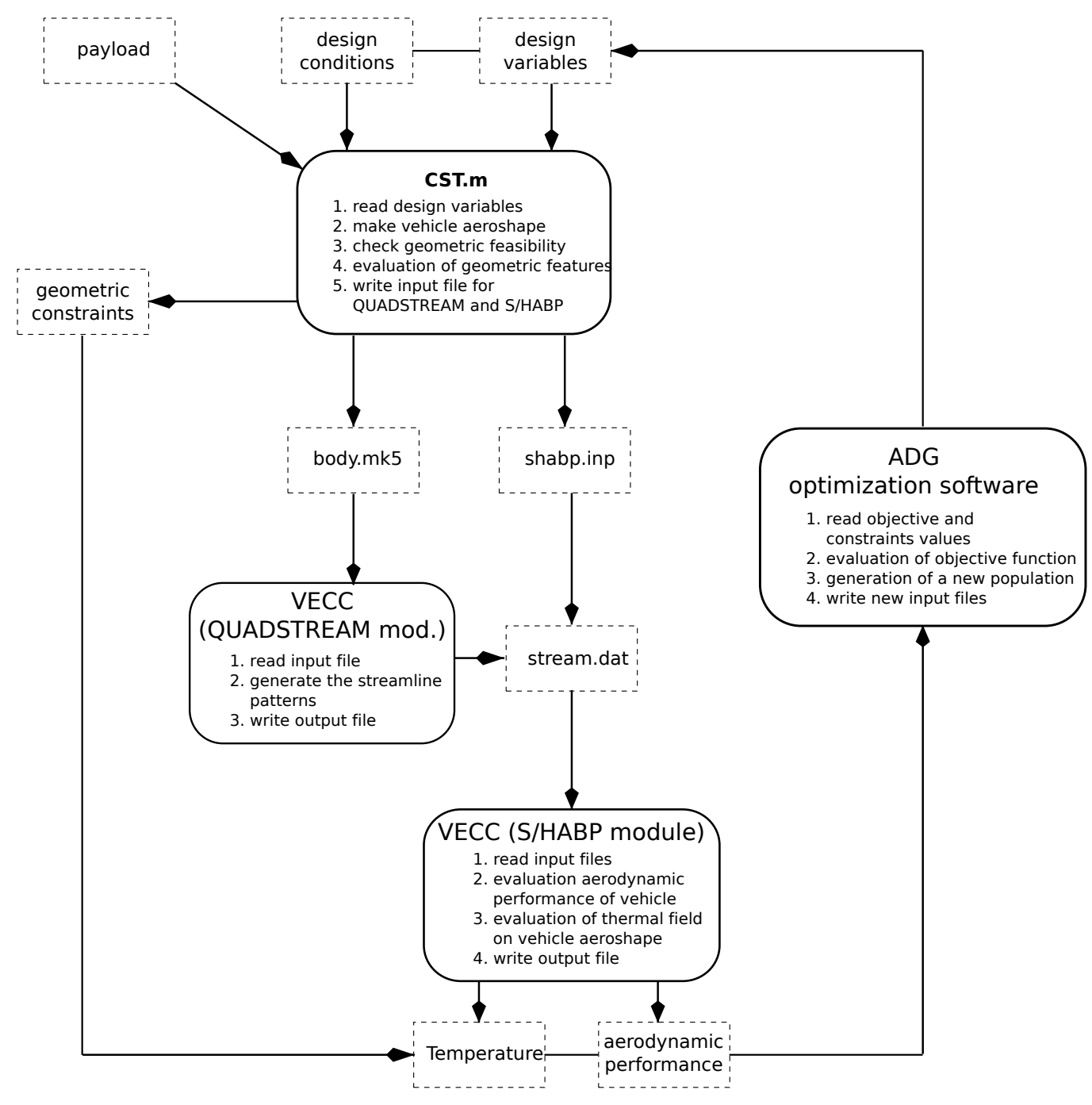

Figure 4: Flow chart of optimization loop for the hypersonic liner optimization problem (Case A and Case B). 
evaluate the constraints. The tool checks the capability of aeroshape of housing the payload (i.e., collision detection) and in output writes a scalar value representative of the violation of this constraint, if any. The collision detection algorithm locates the intersection point between payload and body surfaces, and it also quantifies the amount of payload that sticks out from the fuselage $[34,35]$. The other geometrical features evaluated by this package are: the trailing edge check position, the leading edge radius, and the fuselage nose radius.

The adopted parameterization allows an automatic discrimination of the aircraft architecture. In particular, based on the input variables, the geometry package generates spacecraft fuselage and wing. The fuselage position is fixed while wing can move in streamwise direction according to the value of the scalar translation variable $x_{W}$ (see Fig. 3). The junction between fuselage and wing is managed at the panelization level: the geometry module finds the overlapping panels and computes their intersections. Afterwards, the mesh is regenerated starting from these points, and the part of the wing inside the fuselage is deleted. Hence, when the wing is entirely contained inside the fuselage, only the latter is considered for the optimization. In this way, the design framework is able to optimize the configuration of the spacecraft without using a binary variable (on/off), and topology optimization is achieved. As a result, both winged and wingless configurations are present in the initial population, and the MDO process automatically determines the topology of the optimal aeroshape.

The second step is the calculation of the vehicle surface streamlines through the Quadstream module of VECC code, needed for the viscous analysis [33]. The last package is the aerothermodynamic solver, which reads two input files. The former contains information about the aeroshape mesh and the parameters needed to feed the aerothermodynamic analysis. Once addressed, the aerodynamic performance, the vehicle aeroheating, the values of the temperature peak, the pitching moment $C_{M}$, lift $C_{L}$ and $\operatorname{drag} C_{D}$ coefficients are written to the output file. This allows evaluating the objective function and the violation of constraints, if any. The last step is accomplished by the optimization software. It evaluates the objective function and writes a new input file, where the design variables are reported. This loop is repeated until the objective function reaches the desired value or the optimization loop is converged. 


\begin{tabular}{lc}
\hline Parameters & value \\
\hline Altitude & $30[\mathrm{Km}]$ \\
Density & $0.018\left[\mathrm{Kg} / \mathrm{m}^{3}\right]$ \\
Cruise speed & $2413.65[\mathrm{~m} / \mathrm{s}]$ \\
Mach number & 8 \\
\hline
\end{tabular}

Table 1: Assumed flight conditions for hypersonic cruise.

\begin{tabular}{lcc}
\hline & Case A & Case B \\
\hline Goal & $\min \left(C_{D}\right)$ & $\min \left(C_{D}\right)$ \\
Constraints & $C_{L} \geq 0.034321$ & $C_{L} \geq 0.11095$ \\
& $-0.01 \leq C_{M} \leq 0.01$ & $-0.01 \leq C_{M} \leq 0.01$ \\
& $T_{\max } \leq 2080[K]$ & $T_{\max } \leq 2080[K]$ \\
Collision detection & $\checkmark$ & $\checkmark$ \\
Trailing edge check position & $\checkmark$ & $\checkmark$ \\
\hline
\end{tabular}

Table 2: Parameters for hypersonic cruise optimization problems.

\section{Numerical results}

The objective of the optimization examples hereafter presented is to identify the best aeroshape in terms of minimum drag at the cruise flight conditions listed in Tab. 1, corresponding to two values of the payload mass (Cases $\mathrm{A}$ and $\mathrm{B}$ ), implying different constraint on the lift coefficient $C_{L}$. The other constraints are common between the two cases, namely the trim condition, feasible temperature for the heat shield material and geometric constraints. A summary of the optimization constraints is given in Tab. 2.

The vehicle aeroshape is divided into four parts, and different panel methods and parameters for the viscous analysis are selected for each one (i.e. nose, fuselage, wing, and leading edge of wing). The panel method used by VECC to evaluate the inviscid aerodynamics are reported in Tab.3, whereas the parameters for the viscous analysis are shown in Tab.4. Radiation cooling with emissivity coefficient $\epsilon=0.8$ is assumed at the aeroshape wall and the ideal gas model is used for the airflow. Although sharp leading edges are allowed from the geometrical description, a minimum curvature radius of 0.5 [mm] was set in order to prevent heat flux singularities.

The first geometric constraint is about the aeroshape capability in housing a payload box $2.5[\mathrm{~m}]$ long and $0.5[\mathrm{~m}]$ wide and tall. These constraints are implemented using the collision detection test that verifies if the payload 


\begin{tabular}{lcc}
\hline Zone & Side & Method \\
\hline Nose & windward & Modified Newtonian \\
& leeward & Prandtl-Meyer \\
\hline Fuse & windward & Tangent Cone \\
& leeward & Prandtl-Meyer \\
\hline Leading edge & windward & Modified Newtonian \\
& leeward & Prandtl-Meyer \\
\hline Wing & windward & Tangent Wedge \\
& leeward & Prandtl-Meyer \\
\hline
\end{tabular}

Table 3: Models used for inviscid analysis [33].

\begin{tabular}{lccc}
\hline Component & Fuse/wing & Nose & 2-D swept leading edge \\
\hline Wall condition & radiative & radiative & radiative \\
Boundary layer state & turbulent & turbulent & laminar \\
Skin Friction formula & Schultz-Grunow & Detra-Hildalgo & Lees \\
Heat transfer formula & Flat Plate & Detra-Hildalgo & Beckwith-Gallagher \\
\hline
\end{tabular}

Table 4: Models used for viscous analysis [33].

is contained into the fuselage. In this way it is possible to consider quite complex 3D shapes for the payload. The mass budget is equal to $464[\mathrm{Kg}]$ and $1500[K g]$ for case A and B, respectively. The geometry package detects any collisions and writes a scalar value which quantifies the violation of housing payload constraint. The second geometrical constraint concerns the position of the wing trailing edge, which cannot lie past the fuselage forebody.

Case A optimization relies on CMA-ES algorithms with a population of thirty-two elements. Each individual of population represents a solution of the problem, namely a vehicle configuration and its aeroshape. Figure 5 shows dimensionless drag $\left(C_{D}\right)$ for each population element. The best vehicle aeroshape, according with the design goal reported in Table 2, is shown in Fig. 6. As one can see, the vehicle features a waverider-like aeroshape. In Fig. 7 the pressure coefficient and the heat flux contours on the vehicle aeroshape are shown. As expected, the nose is the most stressed vehicle part from the thermo-mechanical point of view.

Case B optimization problem is addressed by using CMA-ES algorithms with a population of thirty-two elements. In Fig. 8 the trend of the dimensionless drag for each individual is shown. The best aeroshape is that 


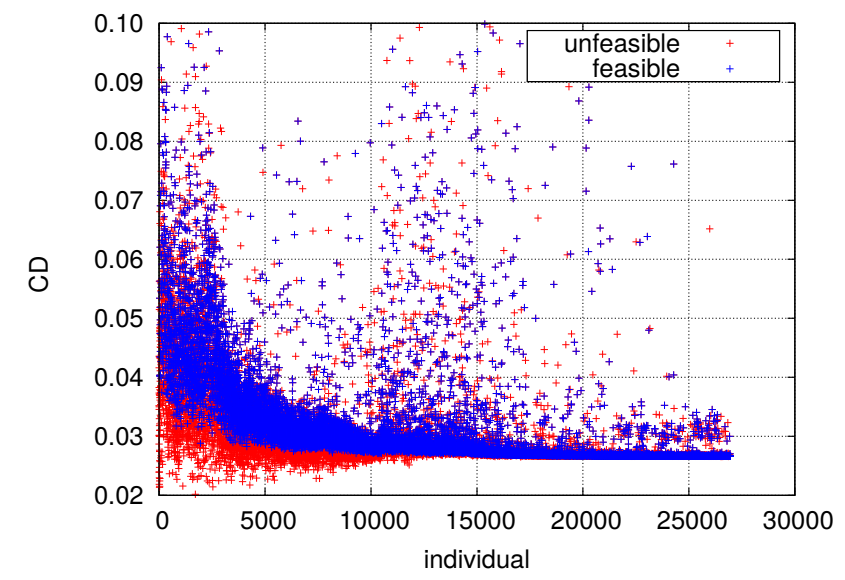

Figure 5: Drag coefficient of vehicle for each individual during the optimization process for Case A problem.

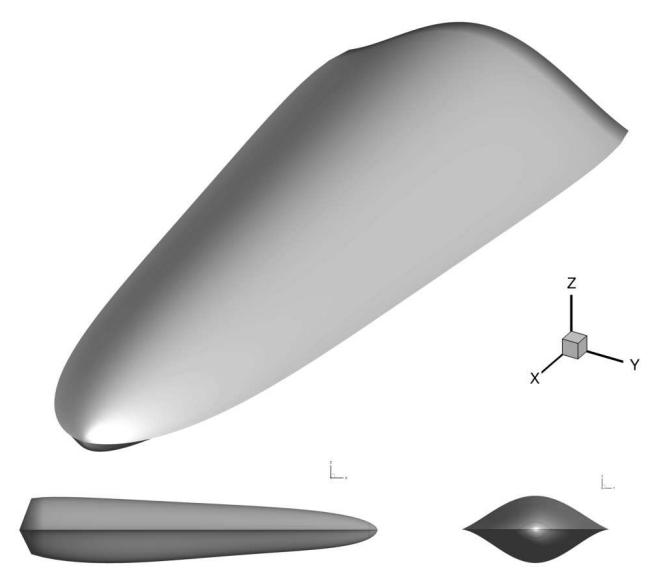

Figure 6: Optimal geometry for Case A problem. 


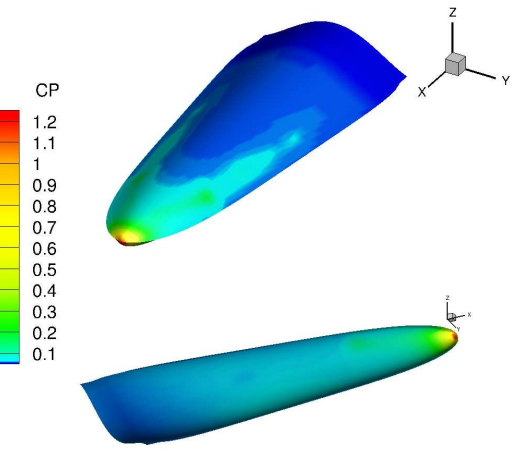

(a)

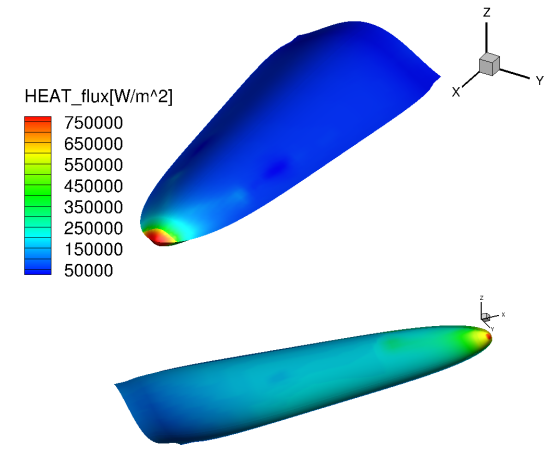

(b)

Figure 7: Wall pressure coefficient (a) and heat flux (b) for Case A optimal vehicle aeroshape.

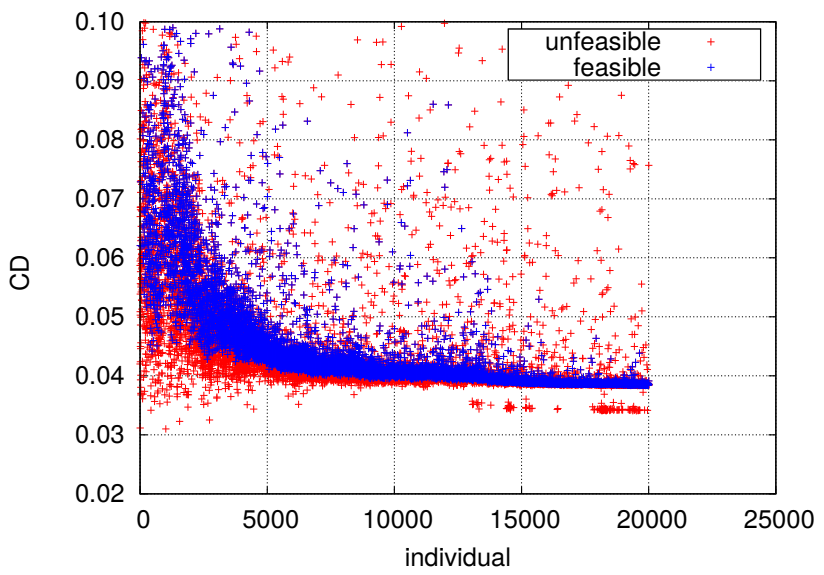

Figure 8: Drag coefficient of vehicle for each individual during the optimization process for Case B problem. 


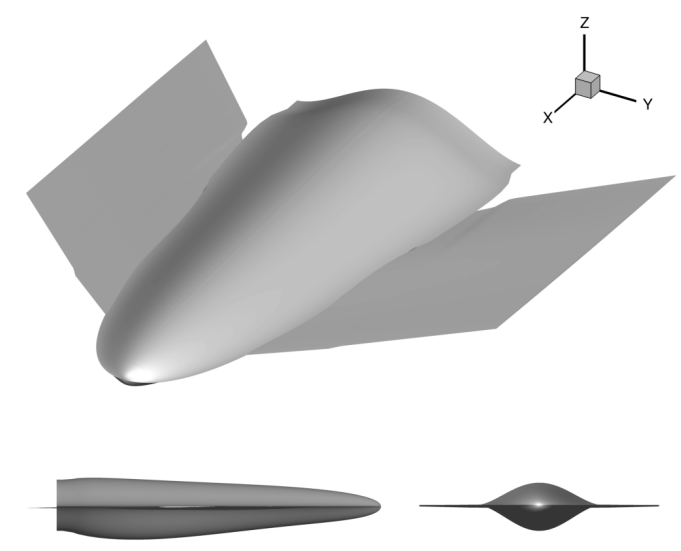

Figure 9: Optimal geometry for Case B problem.

in Fig. 9. In this case, the aerodynamic lift provided only by the fuselage (as in the previous case A) is insufficient to satisfy the vertical equilibrium condition, and a winged-body architecture is suggested and refined by the optimizer. In Fig. 10 the temperature contours on the vehicle aeroshape are shown. As one can see, the peak heating condition takes place on the vehicle nose, although the swept wing leading edge mitigates the temperature on this wing critical part. It is worth noting that although the parameterization allows different sweep angles and taper ratios for the three wing chunks, the computational model favored a flat plate-like configuration, which, at the same time, maximized the L/D and minimized the heat flux in hypersonic conditions.

\subsection{Design results overview}

The main characteristics of the two optimum vehicles are listed in Tab. 7 where the aerodynamic coefficients are evaluated with respect to the vehicle planform area and fuselage length $L$, as references quantities. Please note that $L$ is a design variable, while the planform area and the width are those related to the reference shape. Design results show that, for both cases, a waverider-like aeroshape was proposed by the optimizer, since both problems have the same objective, i.e. achieving minimum drag. The cross sections of the two vehicles at the same $x$ station are shown in Fig. 11. Indeed, in Case A a high aeroshape fineness was provided even though constraints on the payload housing were respected. On the other hand, Case B also features a wing 


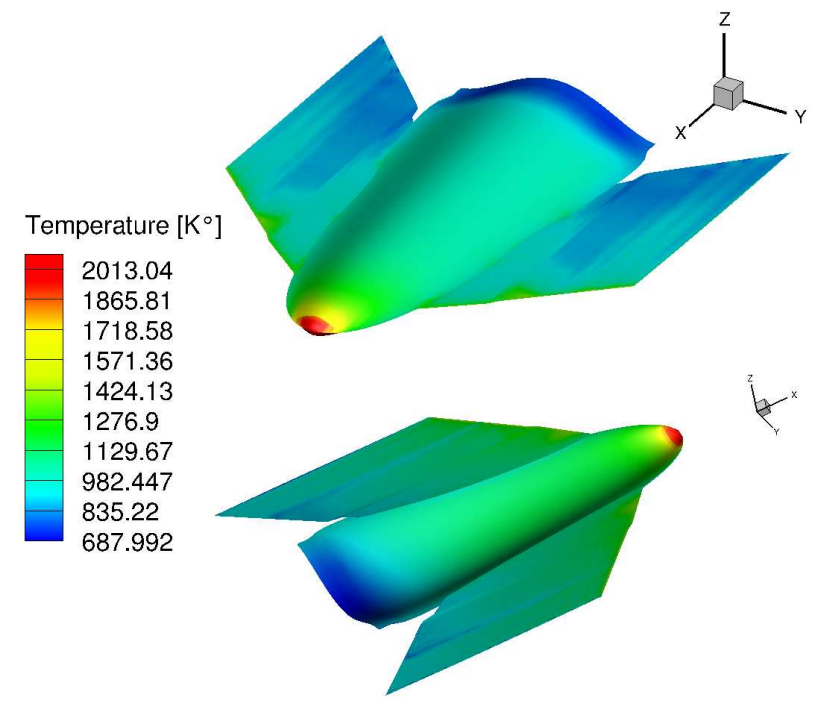

Figure 10: Wall temperature for Case B optimal vehicle aeroshape.

\begin{tabular}{lcc}
\hline Fuse & case A & case B \\
\hline$L[m]$ & 3.50 & 3.49 \\
$A o A[d e g]$ & 2.83 & 2.51 \\
$N C[-]$ & 0.949 & 0.991 \\
$N D 1[-]$ & 0.542 & 0.480 \\
$N D 2[-]$ & 0.105 & 0.154 \\
$A c_{U_{1}}[m]$ & 0.462 & 0.119 \\
$A c_{U_{2}}[m]$ & 0.136 & 0.124 \\
$A c_{L_{1}}[m]$ & 0.044 & 0.028 \\
$A c_{L_{2}}[m]$ & 0.371 & 0.350 \\
$A d_{1}[m]$ & 0.258 & 0.249 \\
$A d_{2}[m]$ & 0.159 & 0.216 \\
$A d_{3}[m]$ & 0.170 & 0.179 \\
$A d_{4}[m]$ & 0.199 & 0.280 \\
\hline
\end{tabular}

Table 5: Fuselage design parameters resulting from the optimization loop. Refer to Section 3.1.2 for nomenclature. 


\begin{tabular}{|c|c|c|}
\hline Wing & case $\mathrm{A}$ & case B \\
\hline$c_{0}[m]$ & - & 1.589 \\
\hline$c_{1}[m]$ & - & 2.018 \\
\hline$c_{2}[m]$ & - & 2.104 \\
\hline$c_{3}[m]$ & - & 2.189 \\
\hline$\lambda_{1}[\mathrm{deg}]$ & - & 38.744 \\
\hline$\lambda_{2}[\mathrm{deg}]$ & - & 52.726 \\
\hline$\lambda_{3}[\mathrm{deg}]$ & - & 42.514 \\
\hline$\alpha[$ deg $]$ & - & 0.439 \\
\hline$\beta[$ deg $]$ & - & 0.000 \\
\hline$y_{1}[m]$ & - & 0.769 \\
\hline$y_{2}[m]$ & - & 0.855 \\
\hline$y_{3}=b / 2[\mathrm{~m}]$ & - & 1.551 \\
\hline$x_{W}[m]$ & - & 0.317 \\
\hline$N 1[-]$ & - & 0.877 \\
\hline$N 2[-]$ & - & 0.987 \\
\hline$A_{U_{0}}[-]$ & - & 0.039 \\
\hline$A_{U_{1}}[-]$ & - & 0.002 \\
\hline$A_{U_{2}}[-]$ & - & 0.010 \\
\hline$A_{U_{3}}[-]$ & - & 0.007 \\
\hline$A_{U_{4}}[-]$ & - & 0.024 \\
\hline$A_{U_{5}}[-]$ & - & 0.071 \\
\hline$A_{U_{6}}[-]$ & - & 0.069 \\
\hline$A_{U_{7}}[-]$ & - & 0.039 \\
\hline$A_{U_{8}}[-]$ & - & 0.041 \\
\hline$A_{L_{1}}[-]$ & - & -0.080 \\
\hline$A_{L_{2}}[-]$ & - & -0.070 \\
\hline$A_{L_{3}}[-]$ & - & -0.089 \\
\hline$A_{L_{4}}[-]$ & - & -0.069 \\
\hline$A_{L_{5}}[-]$ & - & -0.096 \\
\hline$A_{L_{6}}[-]$ & - & -0.096 \\
\hline$A_{L_{7}}[-]$ & - & -0.100 \\
\hline$A_{L_{8}}[-]$ & - & -0.083 \\
\hline
\end{tabular}

Table 6: Wing design parameters resulting from the optimization loop. The results for case A are not shown as the optimal configuration is wing-less. Refer to Section 3.1.1 for nomenclature. 


\begin{tabular}{lcc}
\hline & case A & case B \\
\hline AoA $[\mathrm{deg}]$ & 2.83 & 2.51 \\
Length $(L)[\mathrm{m}]$ & 3.50 & 3.49 \\
Width $[\mathrm{m}]$ & 1.42 & 3.30 \\
$S_{\text {ref }}\left[\mathrm{m}^{2}\right]$ & 3.73 & 4.45 \\
$C_{M}$ & 0.00266 & 0.00104 \\
$C_{D}$ & 0.0179 & 0.0217 \\
$C_{L}$ & 0.0251 & 0.0629 \\
$L / D$ & 1.39 & 2.89 \\
$T_{\max }[\mathrm{k}]$ & 2046.37 & 2016.29 \\
\hline
\end{tabular}

Table 7: Main figures of merit for optimal vehicles.

plane in order to provide the lift/weight balance in this case of higher mass budget $(1500 \mathrm{~kg})$. Anyway, it is worth noting that both aeroshapes suggest a remarkable influence of the Newtonian method (i.e, tangent cone) used in addressing vehicle aerodynamics. For instance, the vehicle configuration is quite similar to an osculatory cone waverider aeroshape, as one can observe by comparing such aeroshapes to those found by Corda and Anderson [36].

To check the accuracy of the VECC results, several CFD analyses have been carried out for Case B optimal shape. CFD simulations are performed with meshes of about $3.5 \times 10^{6}$ cells for the half-body and considering the Eulerian, perfect gas approximation. The specific heat at constant pressure, $c_{p}$, relies on kinetic theory. Seven degree of freedom for the flow molecular species are considered to account for high temperature real gas effects of air. However, dissociation of chemical species may be neglected because the shape is slender and the Mach number is moderate.

On the other hand, the panel code uses a mesh of 3422 panels for the half-fuselage and 7198 for the half-wing. Results comparison for pitching moment coefficient and pressure coefficient distribution is provided in Fig. 12 and Fig. 13, respectively. As one can see, VECC and CFD results compare rather well, thus confirming the reliability of the panel method used in the optimization chain. In particular, Fig. 12 points out that the optimum Case $\mathrm{B}$ aeroshape is statistically stable in longitudinal flight and features a natural trim condition (i.e. $C_{M}=0$ ) at about $\alpha=5$ deg. 


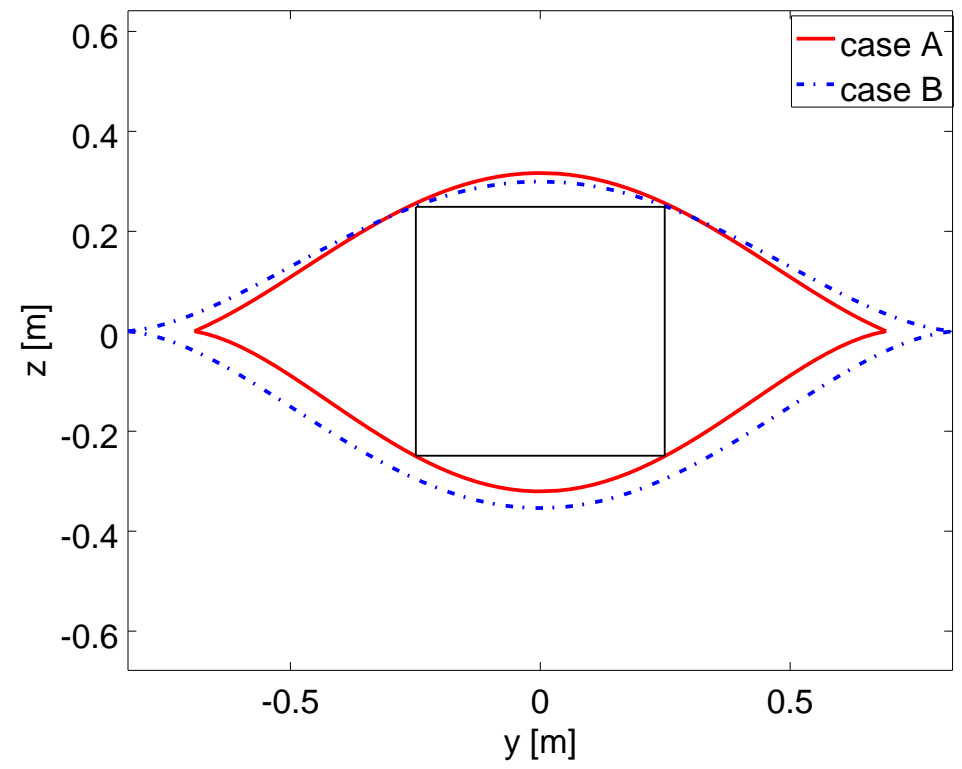

Figure 11: Cross-section shapes of optimal vehicles for Cases A and B with section of payload (solid black line).

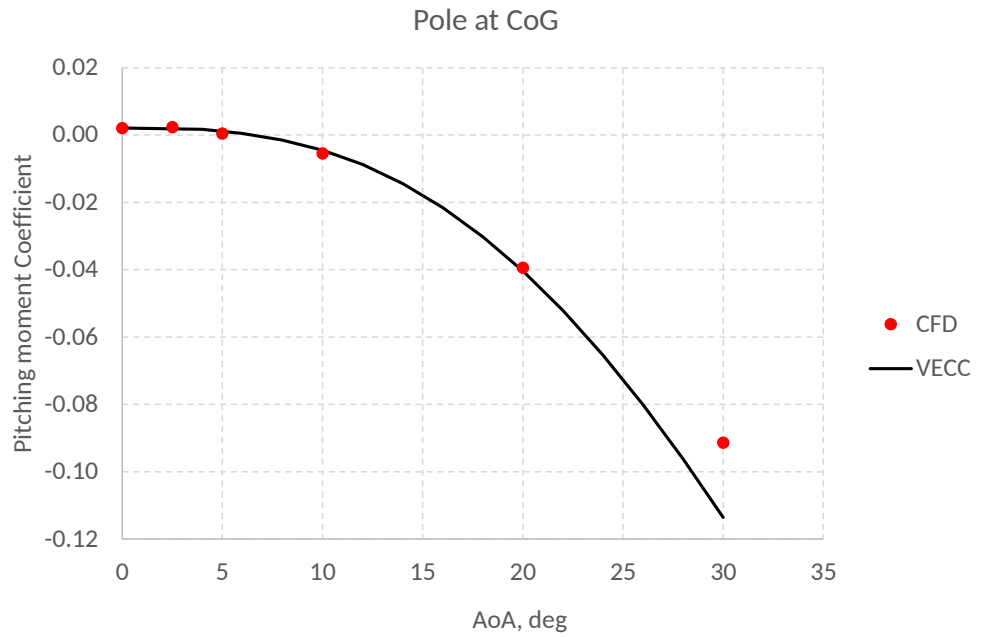

Figure 12: Pitching moment coefficient versus angle of attack: comparison between CFD and VECC results. Pole reduction at vehicle CoG. 


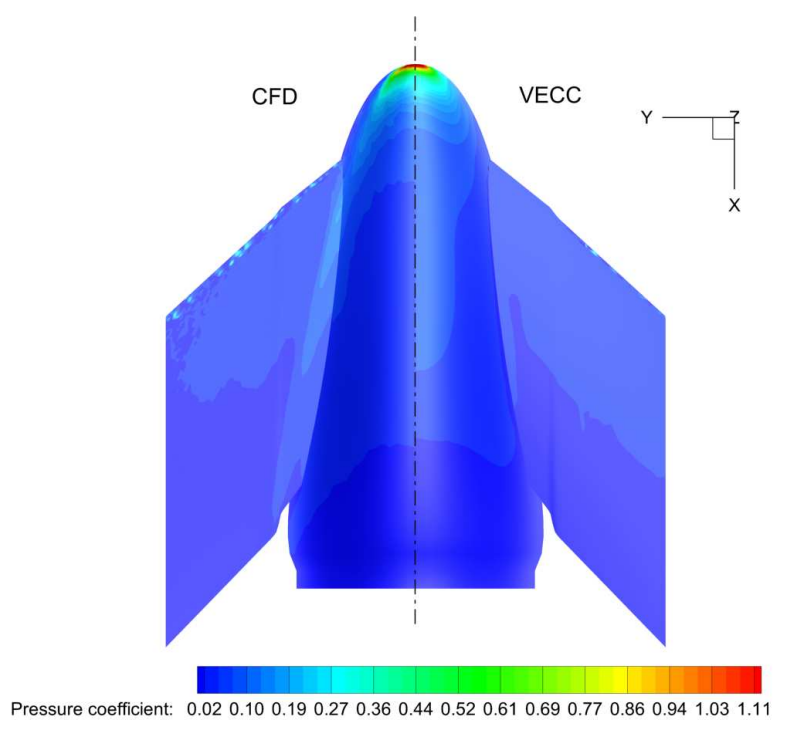

Figure 13: Pressure coefficient on vehicle windside: comparison between CFD and VECC results.

\section{Conclusions}

This paper dealt with the setup of a design optimization framework aimed at the aerodynamic design of hypersonic vehicles. The design environment was conceived in a modular way, so as to allow an easy exchange of both optimization and analysis models. The geometrical parametrization is based on a special CST formulation. This allows efficient description and manipulation of very complex aerodynamic shapes using a reduced number of design parameters. Further, modules have been developed for flight trajectory computation and to address the aerothermal loading environment. This design framework was exploited for the optimization of a hypersonic cruise flying test bed. In particular, a light and a heavy payload design problems were addressed. In the former case, the optimization leads to a configuration similar to those found by many authors in the literature. In the latter case, the optimizer found a winged configuration which has not yet been widely studied. The design results were cross-checked using an Euler flow solver which confirmed the reliability of the analysis chain.

The developed design framework demonstrated interesting design capabilities and promises extension to more challenging and realistic aerothermodynamic design problems, since the present approach allows working si- 
multaneously on both shape and topology of the aircraft. As future work, improvements are planned to extend the design loop by including multiobjective and multi-disciplinary requirements. Forthcoming improvements of the present design framework include upgrade of the MDO capabilities through the inclusion of thermal and structural analysis in the optimization loop, which will allow to account for aerothermal loads (i.e., convective and diffusive heat fluxes) that the spacecraft has to withstand during flight. Further, it is also planned to introduce RANS solvers to explore flight regimes not well described by Newtonian methods. Of course, the challenge will be to preserve the efficiency and the exploring capabilities of our approach. For this purpose, multi-fidelity and surrogate based optimization algorithms will be adopted. Finally, stability and control issues will be addressed to analyze the whole mission design, including subsonic and transonic flight conditions.

\section{REFERENCES}

[1] N. Hansen. The CMA evolution strategy: a comparing review. In J.A. Lozano, P. Larranaga, I. Inza, and E. Bengoetxea, editors, Towards a new evolutionary computation. Advances on estimation of distribution algorithms, pages 75-102. Springer, 2006.

[2] B. Kulfan and J. Bussoletti. "fundamental" parameteric geometry representations for aircraft component shapes. Multidisciplinary Analysis Optimization Conferences. American Institute of Aeronautics and Astronautics, September 2006. doi: 10.2514/6.2006-6948. URL http://dx.doi.org/10.2514/6.2006-6948.

[3] B. Kulfan. A universal parametric geometry representation method "cst". Aerospace Sciences Meetings. American Institute of Aeronautics and Astronautics, January 2007. doi: 10.2514/6.2007-62. URL http://dx.doi.org/10.2514/6.2007-62.

[4] B. M. Kulfan. Universal parametric geometry representation method. J. Aircr., 45(1):142-158, January 2008. ISSN 0021-8669. doi: 10.2514/1.29958. URL http://dx.doi.org/10.2514/1.29958.

[5] M. Y. Wang, X. Wang, and D. Guo. A level set method for structural topology optimization. Comput. Methods Appl. M., 192(1):227 - 246, 2003. ISSN 0045-7825. 
doi: $\quad$ https://doi.org/10.1016/S0045-7825(02)00559-5. URL http://www.sciencedirect.com/science/article/pii/S0045782502005595.

[6] G. Pezzella, M. Marini, M. Cicala, A. Vitale, T. Langener, and J. Steelant. $32^{\text {nd }}$ AIAA aviation (applied aaerodynamic conference). In "Aerodynamic Characterization of HEXAFLY Scramjet Propelled Hypersonic Vehicle”. AIAA 2014-2844, 16 - 20 June 2014, Atlanta, GA.

[7] G. Pezzella, S. van Brummen, and J. Steelant. 8th european symposium on aerothermodynamics for space vehicles. In Assessment of $\mathrm{Hy}$ personic Aerodynamic Performance of the EFTV-ESM Configuration in the Framework of the Hexafly-Int Research Project. European Space Agency, 2-6 March 2015. Lisbon, Portugal.

[8] K.G. Bowcutt, J.D. Anderson, and D. Capriotti. Viscous optimized hypersonic waveriders. In 25th Aerospace Sciences Meeting, Reno, Nevada, January 1987. AIAA.

[9] D. Kuchemann. The aerodynamic design of aircraft. Robert Maxwell, M.C., 1978.

[10] K.G. Bowcutt. Hypersonic aircraft optimization including aerodynamic, propulsion, and trim effects. In Fourth International Aerospace Planes Conference. AIAA, December 1992.

[11] S. Sheffer and G. Dulikravich. Constrained optimization of threedimensional hypersonic vehicle configurations. In 31st Aerospace Sciences Meeting, Aerospace Sciences Meetings. American Institute of Aeronautics and Astronautics, January 1993. doi: 10.2514/6.1993-39. URL http://dx.doi.org/10.2514/6.1993-39.

[12] M. Landon, D. Hall, J. Udy, and E. Perry. Automatic supersonic/hypersonic aerodynamic shape optimization. In 12th Applied Aerodynamics Conference, Fluid Dynamics and Colocated Conferences. American Institute of Aeronautics and Astronautics, June 1994. doi: 10.2514/6.1994-1898. URL http://dx.doi.org/10.2514/6.1994-1898.

[13] A. E. Gentry, D. N. Smyth, and W. R. Oliver. The Mark IV SupersonicHypersonic Arbitrary-Body Program, Volume I - Users Manual. USAF Flight Dynamics Laboratory, 1973. 
[14] F. Ferguson, S. Chandra, I. Blankson, Jr J. Anderson, and A. Kothari. A design method for the construction of hypersonic vehicle configurations. In International Aerospace Planes and Hypersonics Technologies, International Space Planes and Hypersonic Systems and Technologies Conferences. American Institute of Aeronautics and Astronautics, April 1995. doi: 10.2514/6.1995-6009. URL http://dx.doi.org/10.2514/6.1995-6009.

[15] K. G. Bowcutt. Multidisciplinary optimization of airbreathing hypersonic vehicles. J. Propul. Power, 17(6):1184-1190, November 2001. ISSN 0748-4658. doi: 10.2514/2.5893. URL http://dx.doi.org/10.2514/2.5893.

[16] R. Starkey, F. Rankins, and D. Pines. Coupled waverider/trajectory optimization for hypersonic cruise. In 43rd AIAA Aerospace Sciences Meeting and Exhibit, Aerospace Sciences Meetings. American Institute of Aeronautics and Astronautics, January 2005. doi: 10.2514/6.2005530. URL http://dx.doi.org/10.2514/6.2005-530.

[17] A. Ueno and K. Suzuki. Cfd-based shape optimization of hypersonic vehicles considering transonic aerodynamic performance. In 46th AIAA Aerospace Sciences Meeting and Exhibit, Aerospace Sciences Meetings. American Institute of Aeronautics and Astronautics, January 2008. doi: 10.2514/6.2008-288. URL http://dx. doi.org/10.2514/6. 2008-288.

[18] D. Dominic and M. Erwin. Optimization of entry-vehicle shapes during conceptual design. Acta Astronaut., 2014.

[19] M. A. Lobbia. Rapid supersonic/hypersonic aerodynamics analysis model for arbitrary geometries. J. Spacecr. Rockets, 54(1):315-322, September 2016. ISSN 0022-4650. doi: 10.2514/1.A33514. URL http://dx.doi.org/10.2514/1.A33514.

[20] E. Iuliano and D. Quagliarella. Proper orthogonal decomposition, surrogate modelling and evolutionary optimization in aerodynamic design. Comput. Fluids, 84(Supplement C):327 - 350, 2013. ISSN 0045-7930. doi: https://doi.org/10.1016/j.compfluid.2013.06.007.

[21] D. Quagliarella and A. Vicini. A genetic algorithm with adaptable parameters. In 1999 IEEE International Conference On Systems, Man, 
And Cybernetics, Tokyo, Japan, October 1999. Institute of Electrical and Electronic Engineers (IEEE).

[22] R. M. Hicks and P. A. Henne. Wing design by numerical optimization. J. Aircr., 15(7):407-412, July 1978. ISSN 0021-8669. doi: 10.2514/3.58379. URL http://dx.doi.org/10.2514/3.58379.

[23] H. Sobieczkly. Parametric airfoils and wings. Notes on Numerical Fluid Mechanics, 68:71-88, 1998.

[24] S. Painchaud-Ouellet, C. Tribes, J. Trpanier, and D. Pelletier. Airfoil shape optimization using a nonuniform rational b-splines parametrization under thickness constraint. AIAA Journal, 44(10):2170-2178, October 2006. ISSN 0001-1452. doi: 10.2514/1.15117. URL http://dx.doi.org/10.2514/1.15117.

[25] M. Nemec, M. Aftosmis, and T. Pulliam. Cad-based aerodynamic design of complex configurations using a cartesian method. Aerospace Sciences Meetings. American Institute of Aeronautics and Astronautics, January 2004. doi: 10.2514/6.2004-113. URL http://dx.doi.org/10.2514/6.2004-113.

[26] W. Yamazaki, S. Mouton, and G. Carrier. Geometry parameterization and computational mesh deformation by physics-based direct manipulation approaches. AIAA Journal, 48(8):1817-1832, August 2010. ISSN 0001-1452. doi: 10.2514/1.j050255. URL http://dx.doi.org/10.2514/1.J050255.

[27] R. Anderson, G., J. Aftosmis, M., and M. Nemec. Constraint-based shape parametrization for aerodynamic design. In Seventh International Conference on Computational Fluid Dynamics (ICCFDr), Big Island, Hawaii, July 2012.

[28] R. Anderson, G. Adaptive shape parametrization for aerodynamic design. Technical report, NASA, May 2015.

[29] J. Samareh. Aerodynamic shape optimization based on free-form deformation. Multidisciplinary Analysis Optimization Conferences. American Institute of Aeronautics and Astronautics, August 2004. doi: 10.2514/6.2004-4630. URL http://dx.doi .org/10.2514/6.2004-4630. 
[30] D. F. Rogers and J. A. Adams. Mathematical Elements for Computer Graphics. Mc Graw-Hill, 1990.

[31] S. Di Giorgio. An optimization framework for hypersonic vehicle design. Master's thesis, Università degli studi di Roma, "La Sapienza", 2017.

[32] J. A. Fay. Theory of stagnation point heat transfer in dissociated air. Journal of the Aerospace Sciences, 25(2):73-85, February 1958. doi: 10.2514/8.7517. URL http://dx.doi.org/10.2514/8.7517.

[33] A. Burns, K., J. Deters, K., P. Haley, C., and A. Kihiken, T. Viscous Effects On Complex Configurations - VECC software user's manual. McDonnell Douglas Aerospace, 1995.

[34] Nancy M. Amato. Determining the separation of simple polygons. International Journal of Computational Geometry EGamp; Applications, 04(04):457-474, 1994. doi: 10.1142/S0218195994000240. URL https://www.worldscientific.com/doi/abs/10.1142/S0218195994000240.

[35] Sinan Kockara, Tansel Halic, K. Iqbal, Coskun Bayrak, and Richard Rowe. Collision detection: A survey. 2007 IEEE International Conference on Systems, Man and Cybernetics, pages 4046-4051, 2007.

[36] S. Corda and J. Anderson, JR. Viscous optimized hypersonic waveriders designed from axisymmetric flow fields. In 26th Aerospace Sciences Meeting, Aerospace Sciences Meetings. American Institute of Aeronautics and Astronautics, January 1988. doi: 10.2514/6.1988-369. URL http://dx.doi.org/10.2514/6.1988-369. 